\title{
Application of the exp-function method to nonlinear lattice differential equations for multi-wave and rational solutions
}

\author{
İsmail Aslan*†
}

\section{Communicated by S. Georgiev}

In this paper, we extend the basic Exp-function method to nonlinear lattice differential equations for constructing multi-wave and rational solutions for the first time. We consider a differential-difference analogue of the Korteweg-de Vries equation to elucidate the solution procedure. Our approach is direct and unifying in the sense that the bilinear formalism of the equation studied becomes redundant. Copyright ๑ 2011 John Wiley \& Sons, Ltd.

Keywords: exp-function method; differential-difference Korteweg-de Vries equation; lattice differential equations; traveling wave solutions

\section{Introduction}

Nonlinear lattice differential equations (NLDEs), also known as differential-difference equations, are used in distinct branches of nonlinear physical/mathematical sciences. To name a few examples, biophysics, condensed matter physics, mechanical engineering, and fields such as molecular crystals, atomic chains, currents in electrical networks. Their crucial role has motivated scientists to develop many integrable NLDEs [1-3]. Contrary to difference equations that are being fully discretized, NLDEs are semi-discretized with some (or all) of their space variables discretized while time is usually kept continuous. Hence, NLDEs can be assumed as hybrid systems. In recent years, the problem of solving NLDEs analytically has been initiated. Finding special types of exact solutions, such as traveling waves, have been important to understand biological, chemical and physical phenomena governed by NLDEs.

Traveling waves may be coupled with distinct velocities and distinct frequencies. Multi-wave solutions are important because they may sometimes be converted into a single soliton of very high energy that propagates over large domains of space without dispersion. As a result, an extremely destructive wave (tsunami for instance) may be produced. Hence, finding exact and explicit solutions with multi-velocities and multi-frequencies for NLDEs has become an attractive research area nowadays. However, the methods commonly used are usually restricted and cannot be used for numerous realistic scenarios. Hirota's method [4] can be used to search for such solutions if the equations considered can be transformed into a bilinear form. But, there is no guarantee that the bilinear forms are known or exist.

In 2006, He and Wu [5] introduced the basic Exp-function method for finding exact solutions of nonlinear evolution equations (NEEs). Currently, this method has gained much popularity. As a result, the method has been applied to various kinds of nonlinear problems in science and engineering [6-11], and recently more attention is paid to its adaptation, generalization, and extension; for instance, differential-difference equations [12], NEEs with variable coefficients [13], stochastic equations [14], coupled NEEs [15], $n$-soliton solutions for NEEs [16-19], rational solutions for NEEs [20]. It is also worth mentioning that in the comprehensive survey studies [21-23], the basic Exp-function method has been considered as a very reliable technique for solving a wide class of nonlinear problems. The present paper is a worthwhile contribution to this effort as well, because the applicability of the basic Exp-function method to NLDEs for multi-wave and rational solutions still remains an interesting and important unstudied problem.

Our objective in this study is twofold: First, to stress the power of the basic Exp-function method in tackling NLDEs for higher order wave solutions as well as rational solutions. Second, to extend it to a differential-difference Korteweg-de Vries equation for the first time. The remainder of this paper is organized as follows. In Section 2, we state the procedure in brief. In Section 3, we analyze our problem. A conclusion is given in Section 4. 


\section{Methodology}

Let us consider an NLDEs for a function $u_{n}=u(n, t)$ of the form

$$
\frac{\partial u_{n}}{\partial t}=P\left(\ldots, u_{n-r}, u_{n}, u_{n+r}, \ldots\right) \text {, }
$$

where $P$ is a polynomial with constant coefficients and $r$ is fixed (not depending on $n$ ). Equation (1) is discrete in the space variable $n$ and continuous in the time variable $t$. There are no restrictions on the level of shifts or on the degree of nonlinearity in Equation. (1). The basic Exp-function method is based on the assumption that the solutions of Equation (1) can be expressed as

$$
u_{n}=\frac{\sum_{i=0}^{p} a_{i} \exp (i \xi)}{\sum_{j=0}^{q} b_{j} \exp (j \xi)}, \quad \xi=d n+k t+\alpha
$$

where $p$ and $q$ are the positive integers to be determined; $a_{i}, b_{j}, d$, and $k$ are arbitrary constants to be specified; $\alpha$ is an arbitrary phase shift. Substituting the ansatz (2) into Equation (1) and balancing the highest order terms one can determine the constants $p$ and $q$. Clearly, the ansatz (2) corresponds to a one-wave solution of Equation (1). To seek for a multi-wave ( $N \geqslant 2$ ) solution of Equation (1), the ansatz (2) can be modified as follows:

$$
u_{n}=\frac{\sum_{i_{1}=0}^{p_{1}} \sum_{i_{2}=0}^{p_{2}} a_{i_{1} j_{2}} \exp \left(i_{1} \xi_{1}+i_{2} \xi_{2}\right)}{\sum_{j_{1}=0}^{q_{1}} \sum_{j_{2}=0}^{q_{2}} b_{j_{1} j_{2}} \exp \left(j_{1} \xi_{1}+j_{2} \xi_{2}\right)}, \quad \xi_{l}=d_{l} n+k_{l} t+\alpha_{l}, \quad I=1,2,
$$

which corresponds to a two-wave solution of Equation (1)

$$
u_{n}=\frac{\sum_{i_{1}=0}^{p_{1}} \sum_{i_{2}=0}^{p_{2}} \sum_{i_{3}=0}^{p_{3}} a_{i_{1} i_{2} i_{3}} \exp \left(i_{1} \xi_{1}+i_{2} \xi_{2}+i_{3} \xi_{3}\right)}{\sum_{j_{1}=0}^{q_{1}} \sum_{j_{2}=0}^{q_{2}} \sum_{j_{3}=0}^{q_{3}} b_{j_{1} j_{2} j_{3}} \exp \left(j_{1} \xi_{1}+j_{2} \xi_{2}+j_{3} \xi_{3}\right)}, \quad \xi_{l}=d_{l} n+k_{l} t+\alpha_{l}, \quad i=1,2,3,
$$

which corresponds to a three-wave solution of Equation (1), and so on. To obtain a rational solution for Equation (1), the ansatz (2) can be further modified as

$$
u_{n}=\frac{\sum_{i=0}^{p} a_{i}\left(\mu_{1} \exp (\xi)+\mu_{2} \xi\right)^{i}}{\sum_{j=0}^{q} b_{j}\left(\mu_{1} \exp (\xi)+\mu_{2} \xi\right)^{j}}, \quad \xi=d n+k t+\alpha,
$$

where $\mu_{1}$ and $\mu_{2}$ are the two embedded constants. It is obvious that when $\mu_{1}=1$ and $\mu_{2}=0$, the ansatz (5) turns out to be the ansatz (2).

Finally, substituting the ansatze (2)-(5) into Equation (1) leads to nonlinear algebraic systems for the unknown parameters. Solving each resulting system (if possible), one can obtain one-wave, two-wave, three-wave, and rational solutions to Equation (1) assuming that they exist.

\section{Application}

In this section, we generalize our procedure to a differential-difference analogue of the Korteweg-de Vries equation [24, 25] which reads as

$$
\frac{\partial}{\partial t}\left(\frac{u_{n}}{1+u_{n}}\right)=u_{n-\frac{1}{2}}-u_{n+\frac{1}{2}} .
$$

Equation (6) was derived from the Volterra system and related to a sort of ladder circuit [2].

First, we assume that Equation (6) admits a solution in the form

$$
u_{n}=\frac{a_{1} \exp (\xi)}{\left(1+b_{1} \exp (\xi)\right)^{2}}, \quad \xi=d n+k t+\alpha,
$$

which is embedded in the ansatz (2). Substituting (7) into Equation (6) and solving the resultant algebraic system for the unknowns $a_{1}, b_{1}, d$, and $k$, we obtain the solution set

$$
k=\exp \left(-\frac{d}{2}\right)(1-\exp (d)), \quad b_{1}=\exp \left(\frac{d}{2}\right)\left(\exp \left(-\frac{d}{2}\right)-1\right)^{2} a_{1}
$$

which leads to a one-wave solution of Equation (6) as

$$
u_{n}=\frac{\left(\exp \left(\frac{d}{2}\right)-1\right)^{4} a_{1} \exp \left(d n-\exp \left(-\frac{d}{2}\right)(\exp (d)-1) t+\alpha\right)}{\left(\left(\exp \left(\frac{d}{2}\right)-1\right)^{2}+a_{1} \exp \left(d n-\exp \left(-\frac{d}{2}\right)(\exp (d)-1) t+\frac{d}{2}+\alpha\right)\right)^{2}},
$$

where $d, a_{1}$, and $\alpha$ remain arbitrary. 
Second, we suppose that Equation (6) admits a solution of the form

$$
u_{n}=\frac{a_{10} \exp \left(\xi_{1}\right)+a_{01} \exp \left(\xi_{2}\right)+a_{11} \exp \left(\xi_{1}+\xi_{2}\right)+a_{21} \exp \left(2 \xi_{1}+\xi_{2}\right)+a_{12} \exp \left(\xi_{1}+2 \xi_{2}\right)}{\left(1+b_{10} \exp \left(\xi_{1}\right)+b_{01} \exp \left(\xi_{2}\right)+b_{11} \exp \left(\xi_{1}+\xi_{2}\right)\right)^{2}},
$$

where $\xi_{l}=d_{l} n+k_{l} t+\alpha_{l}, I=1,2$. It is obvious that the ansatz (10) is embedded in the ansatz (3). Substituting (10) into Equation (6) and solving the resultant algebraic system for the unknowns $a_{10}, a_{01}, a_{11}, a_{21}, a_{12}, b_{10}, b_{01}, b_{11}, k_{1}, k_{2}, d_{1}$, and $d_{2}$, we obtain the solution set

$$
\begin{aligned}
& k_{1}=\exp \left(-\frac{d_{1}}{2}\right)\left(1-\exp \left(d_{1}\right)\right), \quad k_{2}=\exp \left(-\frac{d_{2}}{2}\right)\left(1-\exp \left(d_{2}\right)\right), \\
& a_{10}=\exp \left(-\frac{d_{1}}{2}\right)\left(\exp \left(\frac{d_{1}}{2}\right)-1\right)^{2} b_{10}, \quad a_{01}=\exp \left(-\frac{d_{2}}{2}\right)\left(\exp \left(\frac{d_{2}}{2}\right)-1\right)^{2} b_{01}, \\
& a_{11}=2 \exp \left(-\frac{d_{1}+d_{2}}{2}\right)\left(\exp \left(\frac{d_{1}}{2}\right)-\exp \left(\frac{d_{2}}{2}\right)\right)^{2} b_{01} b_{10} \\
& b_{11}=\left(\exp \left(\frac{d_{1}}{2}\right)-\exp \left(\frac{d_{2}}{2}\right)\right)^{2}\left(\exp \left(\frac{d_{1}+d_{2}}{2}\right)-1\right)^{-2} b_{01} b_{10}, \\
& a_{21}=\exp \left(-\frac{d_{2}}{2}\right)\left(\exp \left(\frac{d_{1}}{2}\right)-\exp \left(\frac{d_{2}}{2}\right)\right)^{2}\left(\exp \left(\frac{d_{2}}{2}\right)-1\right)^{2}\left(\exp \left(\frac{d_{1}+d_{2}}{2}\right)-1\right)^{-2} b_{01} b_{10}^{2}, \\
& a_{12}=\exp \left(-\frac{d_{1}}{2}\right)\left(\exp \left(\frac{d_{1}}{2}\right)-1\right)^{2}\left(\exp \left(\frac{d_{1}}{2}\right)-\exp \left(\frac{d_{2}}{2}\right)\right)^{2}\left(\exp \left(\frac{d_{1}+d_{2}}{2}\right)-1\right)^{-2} b_{01}^{2} b_{10} .
\end{aligned}
$$

Finally, employing the determined coefficients (11a)-(11f) to (10), we derive a two-wave solution for Equation (6), where $b_{01}, b_{10}$, $d_{1}, d_{2}, \alpha_{1}$, and $\alpha_{2}$ remain arbitrary

For a rational solution, we suppose that Equation (6) admits a solution of the form

$$
u_{n}=\frac{a_{1}\left(\mu_{1} \exp (\xi)+\mu_{2} \xi\right)+a_{0}+a_{-1}\left(\mu_{1} \exp (\xi)+\mu_{2} \xi\right)^{-1}}{b_{1}\left(\mu_{1} \exp (\xi)+\mu_{2} \xi\right)+b_{0}+b_{-1}\left(\mu_{1} \exp (\xi)+\mu_{2} \xi\right)^{-1}}, \quad \xi=d n+k t+\alpha
$$

Following the same procedure, we obtain the solution set of the resultant algebraic system as

$$
k=-\frac{d\left(a_{1}+b_{1}\right)^{2}}{b_{1}^{2}}, \quad a_{0}=\frac{a_{1} b_{0}}{b_{1}}, \quad a_{-1}=\frac{a_{1} b_{0}^{2}}{4 b_{1}^{2}}-\frac{1}{4} a_{1} d^{2}-\frac{1}{4} b_{1} d^{2}, \quad b_{-1}=\frac{b_{0}^{2}}{4 b_{1}}, \quad \mu_{1}=0, \quad \mu_{2}=1,
$$

which leads to a rational solution for Equation (6) as

$$
u_{n}=\frac{a_{1}}{b_{1}}-\frac{b_{1}^{3}\left(a_{1}+b_{1}\right) d^{2}}{\left(b_{0} b_{1}-2\left(a_{1} d\left(a_{1}+2 b_{1}\right) t-b_{1}^{2}((n-t) d+\alpha)\right)\right)^{2}}
$$

where $a_{1}, b_{1}, b_{0}, d$, and $\alpha$ remain arbitrary.

Remark 1

Of course, we could search for $N(\geqslant 3)$-wave solutions for Equation (6) in a parallel manner. However, the calculation procedure becomes tedious and more complicated since more constraint equations need to be satisfied. It is also worth pointing out here that compared with the results of [26], our findings seem to be more general in the sense that they contain more arbitrary parameters. In addition, unlike the procedure of [26], our approach does not require a dependent variable transformation to convert the original equation into an equation in the so-called bilinear form.

\section{Remark 2}

We verified the results of (9), (10), and (14) by back-substitution, this provided an extra measure of confidence in the results. There have been some occasions when solutions obtained by the Exp-function method might be misleading [27-29]. Thus, it is crucial to have a eagle-eyed solving mode when the basic Exp-function method is used.

\section{Conclusion}

We studied a new problem of finding multi-wave and rational solutions to NLDEs via some known generalizations [16-20] of the basic Exp-function method. To achieve our goal, we focused on a differential-difference Korteweg-de Vries equation. We confirmed that the model supports one-wave, two-wave, and rational solutions. We observed that Hirota's dependent variable transformation becomes superfluous in our approach. 


\section{Acknowledgements}

The author thanks the anonymous referees for the valuable and useful suggestions.

\section{References}

1. Toda M. Theory of Nonlinear Lattices. Springer: New York, 1989.

2. Wadati M. Transformation theories for nonlinear discrete systems. Progress of Theoretical Physics Supplement 1976; 59:36-63.

3. Ablowitz MJ, Ladik J. Nonlinear differential-difference equation. Journal of Mathematical Physics 1975; 16:598-603.

4. Hirota R. The Direct Method in Soliton Theory. Cambridge University Press: Cambridge, 2004.

5. He JH, Wu XH. Exp-function method for nonlinear wave equations. Chaos Solitons Fractals 2006; 30:700-708.

6. Mohyud-Din ST, Noor MA, Waheed A. Exp-function method for generalized travelling solutions of Calogero-Degasperis-Fokas equation. Zeitschrift für Naturforschung A-A Journal of Physical Sciences 2010; 65:78-84.

7. Öziş T, Aslan İ. Exact and explicit solutions to the (3+1)-dimensional Jimbo-Miwa equation via the Exp-function method. Physics Letters $A$ 2008; 372:7011-7015.

8. Aslan I. Generalized solitary and periodic wave solutions to a $(2+1)$-dimensional Zakharov-Kuznetsov equation. Applied Mathematics and Computation 2009; 217:1421-1429.

9. Mohyud-Din ST. Solution of nonlinear differential equations by exp-function method. World Applied Sciences Journal 2009; 7:116-147.

10. Aslan I. The Exp-function approach to the Schwarzian Korteweg-de Vries equation. Computers and Mathematics with Applications 2010; 59:2896-2900.

11. Aslan I. Application of the Exp-function method to the $(2+1)$-dimensional Boiti-Leon-Pempinelli equation using symbolic computation. International Journal of Computer Mathematics 2011; 88:747-761.

12. Zhu SD. Exp-function method for the hybrid-lattice system. International Journal of Nonlinear Sciences and Numerical Simulation 2007; 8:461-464.

13. Zhang S. Application of Exp-function method to a KdV equation with variable coefficients. Physics Letters A 2007; 365:448-453.

14. Dai CQ, Zhang JF. Application of He's Exp-function method to the stochastic mKdV equation. International Journal of Nonlinear Sciences and Numerical Simulation 2009; 10:675-680.

15. Yomba E. Application of Exp-function method for a system of three component-Schrödinger equations. Physics Letters A 2009; 373:4001-4011.

16. Marinakis V. The Exp-function method and $n$-soliton solutions. Zeitschrift für Naturforschung A 2008; 63:653-656.

17. Zhang S, Zhang HQ. Exp-function method for $N$-soliton solutions of nonlinear evolution equations in mathematical physics. Physics Letters $A$ 2009; 373:2501-2505.

18. Aslan I. Multi-wave and rational solutions for nonlinear evolution equations. International Journal of Nonlinear Sciences and Numerical Simulation 2010; 11:619-623.

19. Aslan İ. Constructing rational and multi-wave solutions to higher order NEEs via the Exp-function method. Mathematical Methods in the Applied Sciences 2011; DOI: 10.1002/mma.1417.

20. Zhang S. Exp-function method: solitary, periodic and rational wave solutions of nonlinear evolution equations. Nonlinear Science Letters A 2010; 1:143-146.

21. Mohyud-Din ST, Noor MA, Noor KI. Some relatively new techniques for nonlinear problems. Mathematical Problems in Engineering $2009 ; 25$. Article ID 234849.

22. Ylrm A, Hosseini MM, Usman M, Mohyud-Din ST. On nonlinear sciences. Studies in Nonlinear Sciences 2010; 1:97-117.

23. He JH. Some asymptotic methods for strongly nonlinear equations. International Journal of Modern Physics B 2006; 20:1141-1199.

24. Hirota R, Satsuma J. A variety of nonlinear network equations generated from the Bäcklund transformation for the Toda lattice. Progress of Theoretical Physics Supplement 1976; 59:64-100.

25. Hirota R. Nonlinear partial difference equations I: a difference analogue of the KdV equation. Journal of the Physical Society of Japan 1977; 43:1429-1433.

26. Zhang Y, Zhao HQ, Li JB. The long wave limiting of the discrete nonlinear evolution equations. Chaos Solitons Fractals 2009; 42:2965-2972.

27. Aslan I. Comment on: 'New exact solutions for the Kawahara equation using Exp-function method' [Journal of Computational and Applied Mathematics 2009; 233:97-102]. Journal of Computational and Applied Mathematics 2010; 234:3213-3215.

28. Aslan I. Remark on: 'Exp-function method for the exact solutions of fifth order KdV equation and modified Burgers equation' [Applied Mathematics and Computation 2009. DOI:10.1016/j.amc.2009.07.009]. Applied Mathematics and Computation 2010; 217:2912.

29. Aslan I. Comment on: 'Application of Exp-function method for (3+1)-dimensional nonlinear evolution equations' [Computers and Mathematics with Applications 2008; 56:1451-1456]. Computers and Mathematics with Applications 2011; 61:1700-1703. 